Meta

Journal des traducteurs

Translators' Journal

\title{
Index of Words and Topics in Volume 23
}

Volume 23, numéro 4, décembre 1978

URI : https://id.erudit.org/iderudit/003187ar

DOI : https://doi.org/10.7202/003187ar

Aller au sommaire du numéro

Éditeur(s)

Les Presses de l'Université de Montréal

ISSN

0026-0452 (imprimé)

1492-1421 (numérique)

Découvrir la revue

Citer ce document

(1978). Index of Words and Topics in Volume 23. Meta, 23(4), 352-358.

https://doi.org/10.7202/003187ar d'utilisation que vous pouvez consulter en ligne.

https://apropos.erudit.org/fr/usagers/politique-dutilisation/ 


\section{INDEX OF WORDS AND TOPICS IN VOLUME 23}

Abortion, 4, p. 322.

Accountant, 2,167

Accounting clerk, 2, p. 167.

Accountant's cashier, 2, p. 167.

A.C. generator, 3, p. 237.

Active ingredient, 3, p. 196.

Activity, 3, p. 197.

Administrator, 3 , p. 259

Admiralty, 3, p. 260

Adverse reactions, 3, p. 197

Aerodynamic heating, 3, p. 256

Aerodyne, 3, p. 257.

Affect (to), 4, p. 292.

Agent, 3, p. 260.

Aircraft, 3, p. 257.

ALPAC report, 4, p. 316.

Alternate, 4, p. 312 .

Alternatives: greater availability in source language voir poetic translation: alternatives: greater availability in source language

Alternatives: importance of in poetic translation

roir poetic translation: alternatives

Alternator, 3, p. 237.

Anchor rope, 3, p. 231.

Angle shot, 4, p. 314.

Animal breeding, 4, p. 328.

Antifouling, 3, p. 231.

Armature, 3, p. 237.

Armature assembly, 3, p. 237

Armature coil, 3, p. 237.

Armature core, 3, p. 237.

Armature reaction, 3 , p. 237.

Armature shaft, 3, p. 237.

Assistant accountant, 2, p. 167.

Assistant auditor, 2, p. 167.

Assistant baker, 2, p. 167.

Assistant breakfast cook, 2, p. 167

Assistant captain waiter, 2, p. 167.

Assistant convention manager, 2, p. 167.

Assistant gardemanger, 2, p. 167.

Assistant general manager. 2, p. 167

Assistant head butcher, 2, p. 167.

Assistant housekeeper, 2, p. 167.

Assistant roast cook. 2, p. 167.

Assistant sauce cook, 2, p. 167

Assistant storekeeper, 2, p. 167.

Assistant vegetable cook, 2, p. 167.

Auditor, 2, p. 167

Authentic, 3, p. 260.

Background, 4, p. 312

Backstay, 3, p. 234.

Bail, 3 , p. 260

Baker, 2. p. 167

Baker's helper, 2, p. 167

Ballast, 3, p. 233

Balloon. 4, 312

Bankrupcy, 3, p. 260.

Banquet manager, 2. p. 167.

Barmaid, 2, p. 167

Barman, 2, p. 167

Barrister, 3, p. 260.
Barroom staff, 2, p. 167.

Bartender, 2, p. 167.

Bartender's helper, 2, p. 167.

Beam 3, p. 231, 233.

Bellboy, 2, p. 167.

Bellhop, 2, p. 167.

Berth, 3, p. 232.

Bilge pump. 3, p. 234.

Bioavailability, 3. p. 198.

Bird's eye view, 4, p. 314.

Block, 3, p. 234.

Blood-brain barrier. 3, p. 198

Boner, 2. p. 167.

Boom. 3, p. 231.

Boom-vang. 3. p. 233.

Botanical Latin, 2, p. 151.

Bow light, 3, p. 233.

Bow pulpit, 3. p. 231.

Box, 4, p. 313.

Boyle's Law, 3, p. 195.

Brasserie attendant, 2, p. 168.

Breakfast cook. 2, p. 168

Breathalyzer, 3 , p. 226.

Broadcast seeder, 4, p. 328.

Brown shoe, 4, p. 291 .

Brown shoe admiral, 4, p. 291.

Brush, 3, p. 236, 237; 4, p. 313.

Brush gear, 3, p. 237.

Buffet chef, 2, p. 168

Bug. 3. p. 257.

Bulkhead, 3, p. 232.

Bunching onion, 4, p. 328.

Busboy, 2, p. 168.

Butcher, 2, p. 168

Butcher's helper, 2, p. 168.

Cable. 2. p. 160.

Cam shaft. 3, p. 196.

Capacitance, 3, p. 245.

Capacitive reactance, 3 , p. 245.

Capacitor, 3, p. 245.

Captain waiter, 2, p. $168: 3$, p. 246.

Captain waitress, 2, p. 168 .

Caption, 4. p. 312 .

Car attendant. 2, p. 168.

Carhop, 2, p. 168.

Carpenter, 2, p. 168

Cartoon, 4, p. 312.

Cartoonist, 4, p. 312.

Car washer, 2, p. 168.

Case law, 3, p. 260

Cashier, 2, p. 168.

Caterer, 2, p. 168

Catering manager, 2, p. 168

Catford, J.C.. 2. p. 127

Caver, 2. p. 168.

Cellarman, 2, p. 168.

Centreboard, 3, p. 232

Chainplate, 3, p. 231

Character, 4, p. 312.

Characterization. 3. p. 260

Checker, 2, p. 168. 
Checkroom attendant, 2, p. 168

Chief cook, 2, p. 168.

Chive, 4 , p. 328 .

Chock. 3, p. 232

Clamcleat, 3, p. 232.

Class association, 3 , p. 231.

Class boat, 3, p. 231

Cleaner, 2, p. 168

Cleat, 3, p. 234.

Clerical staff, 2, p. 168.

Clew, 3. p. 234

Clew outhaul adjuster, 3, p. 232.

Cliff hanger, 4, p. 312

Close medium shot, 4 , p. 313 .

Close-up, 4, p. 313 .

Club-foosted jib, 3, p. 233.

Coating, 3, p. 196.

Cockpit, 3. p. 232

Cockpit cover, 3 , p. 234

Coffeeman, 2. p. 168.

Coil, 3, p. 245

Collar finisher, 2, p. 168.

Color crayon, 4, p. 313.

Colored inks, 4, p. 313.

Coloring. 4, p. 313.

Colorist, 4 , p. 313

Coloured boot top, 3, p. 232

Colza, 4. p. 328

Coaming, 3, p. 233

Collector brush, 3, p. 236

Collector ring, 3, p. 235

Combustion chamber. 3, p. 256.

Comic artist, 4. p. 312.

Comic book, 4, p. 312.

Comics, 4, p. 312

Comic strip, 4, p. 312.

Common law, 3, p. 258

Common ownership, 3, p. 259.

Commutator, 3, p. 236, 237.

Companionway, 3. p. 232.

Compensation windings, 3 , p. 238

Compound generator. 3. p. 238.

Computational linguistics, 4, p. 316.

Condenser, 3. p. 245.

Conditions, 3 , p. 259

Conductance, specific. 3, p. 245.

Conducting member, 3, p. 236

Conductivity, 3 , p. 244

Conductor, 2. p. 161, 236

Confectionery and pastry cook, 2, p. 168.

Confectionery chief, 2, p. 168.

Constant current, 3, p. 236

Construction of statutes, 3 , p. 260.

Construe. to, 3. p. 260

Continuity, 4, p. 312.

Continuous current, 3, p. 236.

Contract under seal, 3 , p. 258.

Control animals, 3, p. 197.

Controller, food and beverage, 3, p. 247.

Convention manager, 2. p. 168

Convention staff, 2 , p. 168

Cook, 2, p. 168.

Cook's helper, 2, p. 168, 246.

Copy. 3, p. 260

Cord, 2. p. 160

Cradle, 3, p. 231.

Crew, 3, p. 232.

Cruising sailboat, 3, p. 235 .

Cunningham eye, 3 , p. 233 .

Current collecting brush, 3, p. 236.
Cutter, 2, p. 168.

Daily strip. 4 , p. 312

Day-sailer, 3, p. 235.

D.C. generator, 3 , p. 238

Debugging. 3, p. 257.

Deck, 3, p. 234.

Deck fitting. 3, p. 234

Deck house. 3, p. 234

Deed, 3, p. 258.

Delay. 3. p. 260

Delivery boy, 2. p. 168

Depth sounder. 3. p. 234.

Develop, 2, p. 154.

Development, 2, p. 154

Devise and bequeath, I, 3, p. 261

Diachronic translation vesir translation: diachronic

Dialogues, 4. p. 312.

Die, 3, p. 196

Direct current, 3, p. 236

Dishwasher, 2, p. 168.

Disintegration, 3, p. 196

Distance shot, 4, p. 313.

Distribution, 3, p. 198.

Dodger, 3, p. 232.

Doorman, 2, p. 168.

Dosage. 3, p. 197.

Doses. 3. p. 197.

Double blind test, 3, p. 198.

Draft, 3. p. 235.

Dramatic highlight, 4, p. 312.

Draughtmanship, 4, p. 313

Drug. 3. p. 196.

Drug store, 4 , p. 340

Drum-wound armature, 3. p. 238.

Dry sketch. 4, p. 313.

During good behavior, 3, p. 260.

During pleasure. 3. p. 260

Dyestuff, 3, p. 196.

Dynamic equivalence, 3, p. 212 .

Dynamo, 3, p. 238.

Ecoline, 4, p. 313

Eddy current. 3 ,p. 238.

Effective, 3, p. 199

Electric, 3, p. 235

Electric energy, 3, p. 235.

Electric motor. 3, p. 235

Electrical, 3. p. 235

Electrical engineer, 3, p. 235

Electrical engineering. 3. p. 244

Electrician. 2. p. 169

Electrician's helper, 2, p. 169.

Electromagnet, 3 , p. 238.

Electromotive force. 3, p. 239.

Electrotechnics, 3, p. 244.

Elevator mechanic, 2. p. 169.

Elevator operator, 2, p. 169.

EMF, 3, p. 239

Ends, 2, p. 161

Engineer, 2, p. 169

Entremetier, 2, p. $169 ; 3$, p. 246

Equipment storeman, 2, p. 169.

Equitable owner, 3. p. 258

Errand boy. 2, p. 169.

Estate, 3, p. 259.

Evaluation of the translator's work, 3, p. $2 / 2$.

Evidence, 3, p. 260.

Excipient, 3. p. 196

Excitation current, 3, p. 239. 
Exciter, 3, p. 239.

Executor, 3, p. 259.

Fairlead, 3, p. 232

Fantasticks. The, 1, p. 97, 107

Fast food cook, 2, p. 169, 172.

Fast order cook, 2, p. 169, 172.

Feed-hopper, 3, p. 196

Feed-shoe, 3, p. 196.

Fender, 3, p. 232

Fiberglass, 3, p. 232

Field coils, 3, p. 239

Field frame, 3, p. 239.

Field magnet, 3 , p. 245

Field system, 3, p. 245.

Field winding, 3, p. 239, 245

Filler, 3, p. 196.

Filling, 2, p. 161 .

Filling yarns, 2, p. 162.

Final draught, 4, p. 313

Fin keel, 3, p. 234.

Fin-keel model, 3, p. 235.

Fireman. 2. p. 169.

Firing chamber, 3, p. 256

First butcher, 2, p. 169.

First captain waiter, 3, p. 246.

First pastrycook, 2, p. 169

First seamstress, 2, p. 169.

First swing cook, 2, p. 169.

Fittings, 3. p. 231

Flag, 3 , p. 234

Flat-compounded generator, 3, p. 239.

Flatwork operator, 2, p. 169.

Floor pantryman, 2, p. 169

Floor service staff, 2, p. 169

Flotation, 3, p. 232

Flowance, 3, p. 196

Flower attendant, 2, p. 169

Flux lines, 3, p. 235

Food and bar cashier, 2, p. 169

Food and beverage controller, 3, p. 247.

Food controller, 2, p. 169.

Foot, 3, p. 231.

Formality, patterns of in speech behaviour which differ from country to country, 1, p. 12, 14.

Foul weather gear, clothing, 3 , p. 232

Frame, 4, p. 313

Framing, 4. p. 313

Free air, 3, p. 256.

Free atmosphere, 3, p. 256

Free-board 3, p. 233.

Freedom of choice in translating, 3, p. 211

From time to time. 3, p. 261

Front desk, 2, p. 169

Front frame, 3, p. 239.

Front office, 2, p. 169.

Front office cashier, 2, p. 169

Frosted acetate, 4, p. 313.

Fruit growing, 4, p. 330 .

Frustration of adventure, 3, p. 259.

Fry cook, 2, p. 169.

Full-figure shot, 4, p. 313.

Full páge strip, $4, p .312$

Fully automatic high-quality translation, 4, p. 316.

Gag, 4, p. 312.

Gag strip, 4, p. 312

Gallery. 3, p. 232

Garage attendant, 2, p. 169

Garage cashier. 2. p. 169
Garage mechanic, 2, p. 169

Garage supervisor, 2, p. 169

Garbage attendant, 2, p. 169.

Gardemanger, 2. p. $169 ; 3$, p. 246.

Gel-coat. 3, p. 233.

General cterk. 2, p. 169.

General manager, 2, p. 169.

Generator. 3, p. 235. 239.

Genoa, 3, p. 233

Gimballed, 3, p. 233.

Gooseneck, 3, p. 235.

Greek, 2, p. 148

Greek and Latin quotations, 2. p. 148.

Green onion, 4, p. 328 .

Grillroom cook, 2, p. 169; 3, p. 246.

Gunwhale, 3, p. 234

Half page strip, 4, p. 312 .

Halyard, 3, p. 232

Hand ironer, 2, p. 170

Handyman, 2, p. 170.

Hanging locker, 3, p. 234.

Hardy, 4, p. 328

Hatch, 3, p. 232

Hayard rope tail, 3 , p. 232.

Head. 3. p. 234. 235.

Head baker, 2. p. 170

Head bartender, 2. p. 170

Head bellboy, 2, p. 170.

Head bellhop, 2, p. 170

Head busboy, 2, p. 170

Head butcher, 2, p. 170

Head carpenter, 2, p. 170.

Head cellarman, 2, p. 170

Head fry cook, 2. p. 170.

Head gardemanger, 2. p. 170

Head housekeeper, 2, p. 170

Head inspector, 2, p. 170.

Head linenkeeper, 2, p. 170.

Headliner, 2, p. 235.

Head maître d'hôtel, 2, p. 170.

Head maître D, 2, p. 170

Head painter, 2, p. 170 .

Head pantryman, 2, p. 170

Head payroll clerk, 2, p. 170.

Head porter, 2, p. 170.

Headroom, 3, p. 233.

Head roast cook, 2, p. 170

Head sauce cook, 2, p. 170

Head scrubber, 2. p. 170.

Headstay, 3, p. 232

Head stillroom girl, 2 p. 170

Head upholsterer, 2, p. 170.

Head swing cook, 2, p. 170.

Head vegetable peeler, 2, p. 170

Head washman, 2, p. 170.

Heating, 3, p. 244

Heavier-than-air-aircraft, 3, p. 257

Heteropolar alternator, 3, p. 240.

Hiking strap, 3, p. 234.

Hinged mast step, 3, p. 232.

Homer, 1, p. 8, 15, 16.

Homopolar alternator, 3 , p. 240

House detective, 2, p. 170.

House doctor, 2, p. 170.

Housekeeper, 2, p. 170; 3, p. 247.

House nurse. 2. p. 170

House officer, 2, p. 170

House physician. 2. p. 170

Housing, 3, p. 240. 
Hull, 3, p. 232.

Humour strip, 4, p. 312.

Iceman, 2, p. 170

Illustrated weekly, 4, p. 312

Inboard motor, 3 , p. 233

Incidence, 4 , p. 322

Indeterminary of translation, 3, p. 211, 213.

Individual doses, 3, p. 197

Induced, 3, p. 236.

Inductance, 3, p. 245

Inductor, 3, p. 245.

Industrial park, 2 , p. 156

Infant, 3, p. 260

Inked, 4, p. 313

Inker, 4, p. 313.

Insert, 4 , p. 313

Inspector, 2, p. 170

Insulation, 3 , p. 245

Interlaced winding, 3, p. 240

Interlingual transfer process, 4, p. 316

Interpoles, 3, p. 240

Invention, 4, p. 314

lroner, 2, p. 170

Israel Association of Applied Linguistics, 2, p. 127.

Jacket. 3, p. 244

Janitorial staff, 2, p. 171

Jib, 3, p. 233.

Jib hank, 3, p. 231

Jiffy reefing, 3, p. 234

Joint ownership, 3, p. 259.

Jurisprudence, 3 , p. 260

Justice (Mr.), 3, p. 260.

Keelboat, 3, p. 234.

Keel- C/B, 3, p. 235

Keel-centreboard model, 3, p. 235

Kinetic heating, 3, p. 256.

Kitchen chef, 2 , p. 17 I.

Kitchen clerk, 3, p. 246

Kitchen fireman, 2, p. 171.

Kitchen foreman, 2, p. 171 .

Kitchen hand, 2, p. 168,171

Kitchen helper, 2, p. 171

Kitchen steward, 2, p. 171.

Knee: shot, 4, p. 313

Laborer, 2, p. 171.

Language, emotional charge of in poetry, 1, p. 7,8, 14.

Lap winding, 3, p. 240.

Latin, 2 p. 148 .

Latin idioms, non-classical, 2, p. 150.

Laundry forelady, 2, p. $17 i$.

Laundryman, 2, p. $171,173$.

Layout, 4 , p. 314

Leach, 3, p. 232

Left hand rule, 3 , p. 240.

Legal owner, 3, p. 258.

Lencht overall, $3, p, 233$

Lenght water-line, 3, p. 233.

Lettering, 4, p. 313.

Lettering man, 4 , p. 313

Lifeline, 3, p. 232.

Liftman, 2. p. 171

Ligant, 3, p. 196

Line, 3 , p. 233

Linenkeeper, 2, p. 171.
Linguistics and translation theory, 3, p. 211.

Literal translation, 1, p. 37, 38. Voir aussi traduction littérale

L.O.A., 3 , p. 233.

Load circuit, 3, p. 236.

Lobbyman, 2, p. $171 ; 3$, p. 247.

Local opinion, 2 , p. 166.

Locker, 3, p. 232.

Locksmith, 2, p. 171.

Long shot, 4, p. 313

Loop, 3, p. 240 .

Lower shroud, 3, p. 231.

Luff, 3. p. 232.

L.W.L., 3, p. 233

Machine-aided translation, 4 , p. 317.

Machine translation, 4, p. 316

Magnetic field, 3, p. 235,240

Magnetic tape, 3, p. 231 .

Magneto, 3, p. 241.

Magnetoelectric generator, 3, p. 241

Maid, 2, p. 171

Mail clerk. 2, p. 171.

Mail girl, 2, p. 171

Main cabin, 3, p. 232

Main sail, 3 , p. 233

Mainsail cover, 3, p. 231

Mainsail slide, slug, 3, p. 232.

Mainsheet. 3, p. 233.

Mainsheet traveller track, 3, p. 234.

Maitre D, 2, p. 171 .

Maitre d'hôtel, 2, p. 171.

Manager, 2, p. 171

Mandrail, 3, p. 233

Manufacturer, 3, p. 244.

Marginal ulcer, 3, p. 195.

Marine toilet. 3, p. 235.

Master, 4, p. 313 .

Master of the rolls, 3, p. 260

Mast grounded to keel, 3, p. 233.

Masthead rigged sloop, 3, p. 234

Mast lowering equipment, 3. p. 232

Medieval poetry, 1, p. 37,46

Medium close shot, $4,0.313$

Medium shot. 4. p. 313 .

Member of parliament, 3, p. 260.

Messenger boy, 2, p. 171 .

Metabolite, 3, p 199.

Metaliterature, 3, p. 218.

Metaphor: definition, 1, p. 14.

Metaphor: translation of its emotional charge in poetry, 1, p. 15, 19

Mid shot, 4, p. 313 .

Miscarriage, 4 , p. 322

Mixing, 3, p. 196.

Mooring line, 3, p. 231

Multiple translations, 1, p. 96.

Multipole alternator, p. 241.

Mutual inductance, 3 , p. 245 .

NAD, 3, p. 195

Narration, 4, p. 312 .

Necessaries, 3, p. 259

Neutral plane, 3, p. 241.

Night cleaner, 2, p. 171.

Night manager, 2, p. 17 !

Nominal voltage, 3, p. 244

Non appreciable disease, 3, p. 195.

Non skid, 3, p. 231.

Notary legal. 3 , p. 260. 
Obviousness, 3, p. 260.

Occupational disease, 4, p. 322

Occupational injury, 4, p. 322.

Oiler, 2, p. 171.

One-design, 3, p. 233.

Open narrative, 4, p. 312.

Outboard motor, 3, p. 233.

Outboard motor bracket, 3, p. 232.

Overlay, 4, p. 313.

Ownership, 3, p. 260.

Page, 4. p. 312 .

Painter, 2, p. 172.

"Palamon and Arcite", 3, p. 200.

Panning shot, 4, p. 314

Pantry, 2, p. 172 .

Pantryman, 2, p. 172

Parallel, 4, p. 312

Parking, 4 , p. 340

Pastry chef, 2, p. 172.

Payroll clerk, 2, p. 172

Pen, 4, p. 313

Peptic ulcer, 3, p. 195.

Permanent magnet, 3, p. 241.

Personal property, 3 , p. 259.

Personal representative, 3, p. 259.

Personalty, 3, p. 259.

Pharmacogenetics, 3, p, 197.

Parmacokinetics, 3. p. 195.

Photograph (to) down to size. 4, p. 313.

Photogravure, 4, p. 313.

Picks, 2, p. 161.

Pigtail. 3, p. 241

Placental barrier, 3, p. 198.

Plasterer, 2, p. 172

Plebiscite, 2, p. 164

Plot, 4 , p. 312

Plumber, 2, p. 172

Plumber's helper, 2, p. 172.

Poetic translation: alternatives, 1, p. 7, 14, 19.

Poetic translation: alternatives: greater availability

in source language, 1, p. 12, 14

Poetic translator: skills required, 1, p. 86

Poetry, criterion of, 1, p. 7.

Pole pieces, 3, p. 241

Polisher, 2, p. 172

Porter, 2, p. 172.

Positive print, 4, p. 313.

Potentialization, 3, p. 199

Potentiation, 3, p. 199

Potwasher, 2, p. 172.

Press operator, 2, p. 172

Prevalence. 4, p. 322

Primary care physician, 4 , p. 322

Primary motor, 3, p. 241.

Process of translation, 3, p. 212.

Progression, 3, p. 199

Property, 3 , p. 260

Property development, 2, p. 155.

Prose translation, 1, p. 38, 39, 44. Voir aussi traduction de vers en prose

Psychological discovery, 4, p. 315.

Psychological invention, 4, p. 315.

Punch, 3, p. 196; 4, p. 312.

Punctuation, lack of in medieval poetry, 1, p. 39.

Qualification, 3, p. 260.

Quick release shackle, 3,p. 233 .

Quick service cook, 2, p. 172.
Radical translation, 3, p. 213.

Raised deck, 3, p. 233.

Rape, 4. p. 328 .

Rapeseed, 4 , p. 328

Rated-voltage, 3, p. 244.

Real estate, 2, p. 154; 3, p. 259.

Realty, 3, p. 259.

Rear frame, 3, p. 241.

Receiving clerk, 2, p. 172.

Receptionist, 2, p. 172

Rectify (to), 3, p. 236.

Red pepper, 4, p. 328.

Reefing claw ring, 3, p. 232.

Referendum, 2, p. 164

Refuse collector, 2, p. 169, 172.

Release, 3, p. 198.

Reliability, 3, p. 197

Relief, 3, p. 246

Relief clerk, 2, p. 172.

Relief staff, 2,p. 172.

Remote metering, 3, p. 256.

Resistance, 3. p. 245 .

Resistor, 3, p, 245.

Restroom attendant, 2. p. 172

Reverse angle shot, 4, p. 314

Rhyme, 1, p. 38.

Rhythm, 1, p. 38 . Voir aussi rythme, préservation de en traduction.

Ring-wound armature, 3, p. 241.

Ripple, 3, p. 241.

Roast cook. 2, p. 172.

Room maid, 2, p. 172.

Room service staff, 2, p. 172.

Rope, 3, p. 232.

Rotating-armature alternator, 3, p. 241

Rotating field alternator, 3, p. 242.

Rotor, 3 , p. 242

Rough sketch, 4, p. 313.

Rubbing strake, 3, p. 233.

Rubrail, 3, p. 233.

Rudder. 3. p. 234.

Running rigging, 3. p. 233.

Safety, 3, p. 197.

Sail area, 3, p. 234

Salient-pole rotor, 3, p. 242.

Sanitary engineer, 2, p. 169, 172.

Sauce cook, 2, p. 172.

Script, 4, p. 3I 2

Scrubber, 2, p. 172

Seamstress, 2, p. 172.

Segment, 3, p. 236.

Self-bailing. 3 , p. 231

Self-contained portable toilet, 3, p. 235.

Self-excited generator. 3 , p. 242.

Self inductance, 3, p. 245.

Semantic mapping, 2, p. 127.

Senior accountant, 2, p. 172.

Senior payroll clerk, 2, p. 172.

Separately excited generator, 3. p. 242.

Serial strip, 4 , p. 312 .

Series field-winding. 3, p. 242.

Series generator, 3. p. 242

Sheath, 3, p. 244

Sheerline, 3, p. 235

Sheet, 3. p. 232.

Sheet frosted acetate. 4, p. 313.

Sheet of clear acetate, 4, p. 313

Shoal-draft model, 3, p. 235.

Short order cook, 2, p. 172. 
Shroud, 3. p. 233

Shunt field-winding, 3, p. 243

Shunt generator, 3, p. 243

Side effects, 3, p. 197.

Simple contract, 3 , p. 258

Simple contract with consideration, 3, p. 258.

Simplified narration, 4, p. 312

Sine wave, 3, p. 243.

Single-phase alternator, 3, p. 243.

Slicer, 2, p. 168, 173

Slicling brush, 3, p. 236.

Sliding contact, 3, p. 236

Slicling hatch cover, 3, p. 231.

Slip ring, 3, p. 235.

Sloop, 3, p. 234.

Smooth-pole rotor, 3, p. 243.

Snap shackle, 3, p. 233.

Soda fountain keeper, 2, p. 173.

Soft drink attendant, 2, p. 173.

Soil management, 4 , p. 330 .

Solder (to). 3, p. 244

Solicitor, 3, p. 260.

Solubilized state. 3, p. 198

Solvent, 3, p. 196.

Source language. 1, p. 12. Voir aussi langue de départ.

Spar, 3, p. 232

Specialty contract, 3 , p. 258

Specific conductance, 3, p. 245.

Speculative builder. 2. p. 155.

Spinnaker, 3, p. 234.

Spinnaker pole, 3, p. 234.

Spontaneous abortion, 4 , p. 322

Spray hood, 3, p. 232.

Spreader, 3, p. 231

S/S. 3, p. 233.

Staff cook, 3, p. 246

Staff waiter, 2, p. $173 ; 3$, p. 246

Staff waitress, 2, p. $173 ; 3$, p. 246.

Stainless steel, 3 , p. 233.

Stanchion, 3, p. 232.

Standing rigging. 3 . p. 323

Stationary-armature alternator, 3, p. 243.

Stator, 3. p. 243.

Stem. 3, p. 232.

Stenographer. 2, p. 173.

Stern rail. 3 , p. 231 .

Steward, 2, p. 173.

Stillroom girl, 2, p. 173.

Stillroom staff, 2. p. 173

Storna ulcer. 3, p. 195.

Storekeeper, 2. p. 173.

Storm jib. 3 , p. 235.

Storyboard, 4, p. 312.

Story line, 4, p. 312 .

Stowage, 3, p. 234.

Strain. 3, p. 197.

Strip, 4, p. 312

Studies, 4, p. 313

Subdivide, 2. p. 156.

Subdivider. 2. p. 155

Sunday pages, 4, p. 312

Supreme Court. 3. p. 260.

Sustained release, 3, p. 198.

Swing cook, 2, p. $173 ; 3$, p. 246

Switch. 3, p. 236.

Switchboard operator, 2. p. 173

Synergism, 3, p. 199.

Synopsis, 4, p. 312.

Systran, 4, p. 317.
Tabletting mass, 3, p. 196.

Tack, 3, p. 234.

Tackle, 3, p. 234.

Tape, magnetic, 3, p. 231

Target language, 1, p. I2 Voir aussi langue cible, langue d'arrivée.

Teak. 3. p. 234.

Telemetering, 3, p. 256

Telemetry, 3, p. 257

Telephone operator, 2, p. 173.

Telltail, 3, p. 234.

Telltale, 3, p. 234

Temperature rise, 3 , p. 244

Teratogenic effects, 3, p. 199.

Terms and conditions of the contract, 3, p. 261.

"The Knight's Tale ", 3, p. 200

Thought balloon, 4, p. 312

Thread. 3, p. 244 .

Three-phase alternator, 3, p. 243

Tiller, 3. p. 231.

Tiller extension. 3, p. 231

Time and place, 3, p. 261

Tinsmith, 2, p. 173.

Tissue culture, 3, p. 197.

Toe rail, 3, p. 231 .

Topping lift, 3, p. 231.

Topsides, 3, p. 231.

Tort, 3, p. 260

Tort feaser, 3, p. 260.

Total doses, 3. p. 197

Tracing paper, 4, p. 313.

Trailer, 3, p. 234.

Translatics, 3, p. 212

Translation and value judgment, 3, p. 211.

Translation by paraphrase, 3, p. 200.

Translation: diachronic, 1, p. 15. 16

Translation equivalence, 2 , p. 127.

Translation of plays on words, 1, p. 86, 96.

Translatology, 3, p. 212.

Transliteration, 2, p. 149.

Transom, 3, p. 234.

Travelling, 4, p. 313

Trespass, 3, p. 260.

Trespass to goods, 3 , p. 260.

Trunk-cabin, 3, p. 234.

Turbo-generator. 3. p. 243

Turnbuckle, 3 , p. 234.

Two-burner stove. 3, p. 234.

Two-phase alternator, 3, p. 244.

Two-pole generator, 3, p. 244.

Ulcer, marginal, 3, p. 195

Ulcer, peptic, 3. p. 195

Ulcer, stoma, 3 p. 195

Uniform room girl, 2, p. 173.

Upholsterer. 2, p. 173.

Upper shroud, 3, p. 233.

Utilityman, 2. p. 170,173

Valet, 2, p. 173.

Valet service attendant. 2. p. 173.

Vegetable cook, 2, p. 173; 3, p. 246.

Vegetable peeler, 2 , p. 173.

Verse translation, i, p. 38, 39. Voir aussi traduction d'un poème en vers.

Vertical clearance, 3, p. 234.

Vetch, 4 , p. 328

Virgil, 1, p. $8,9,30$

Visual place, 4, p. 314. 
Waiter, 2 p. 173

Waitress, 2, p. 173

Wall washer, 2, p. 173

Warp. 2, p. 161

Warp threads, 2, p. 162 .

Warranties, 3, p. 259

Washman, 2, p. 173

Washman's helper, 2, p. 173

Watercolour, 4, p. 313.

Waterline, 3, p. 232

Wave winding, 3 , p. 244

Wear resistance, 3 , p. 196.

Week-end, 4 , p. 340 .

Weft, 2, p. 161 .

Weld (to), 3, p. 244

Wheel steering, 3. p. 231

Whisker pole, 3 , p. 234
Wicke, 4, p. 328

Winch, 3 , p. 235

Wind indicator, 3, p. 233.

Wine steward, 2, p. 173.

Wire, 2, p. 159: 3, p. 244.

Wire-rope, 3, p. 231

Working jib, 3, p. 233

Working sails, 3, p. 235

Workman, 2, p. 171, 173.

Worm's eye view, 4, p. 314.

Writer, 4. p. 312

Wye connection. 3, p. 244.

Zoom, 4, p. 314

Zoom in 4, p. 314

Zoom out, 4, p. 314 